\title{
A IMPORTÂNCIA DO ERGODESIGN NA AVALIAÇÃO DE CD-ROM SOBRE DENGUE E DOENÇA DE CHAGAS NA EDUCAÇÃO EM SAÚDE
}

\author{
THE IMPORTANCE OF ERGODESIGN IN THE ASSESSMENT OF CD-ROMS ABOUT DENGUE FEVER \\ AND CHAGAS DISEASE IN HEALTH EDUCATION
}

\author{
Denise Nacif Pimental \\ Heloisa Maria Nogueira Diniz² \\ Manuel de Almeida Magalhães Andrade 3 \\ Patrícia Rezende de Oliveira 4 \\ Juliana Ferreira da Silva ${ }^{5}$ \\ João Carlos Pinto Dias6 \\ Virgínia Torres Schall7 \\ Robson Santos 8
}

Resumo Na interseção entre saúde, educação e o controle e prevenção de doenças infecciosas e parasitárias, consideram-se os conceitos e práticas de ergodesign como elementos fundamentais para geração de interfaces de materiais para aprendizagem interativa em saúde, que reflitam a relação entre usuário, tarefas e ambientes. Relata-se a avaliação de CD-ROM a partir de princípios do ergodesign, com vistas a colaborar para o acesso e disseminação da informação em saúde e auxiliar na aprendizagem interativa para profissionais de saúde de nível médio e superior. Para tal, realizou-se levantamento de 21 materiais digitais de instituições internacionais e nacionais sobre as duas doenças. Os materiais foram avaliados através de uma lista de verificação: ergolist. Verificou-se que os materiais avaliados não atendem plenamente aos critérios de avaliação adotados. A avaliação e produção de tecnologias de informação e comunicação, como CD-ROM educativos e interativos, a partir dos princípios do ergodesign podem servir como instrumento facilitador na disseminação da informação e redução de fronteiras entre educação e saúde.

Palavras-chave educação em saúde; dengue e doença de Chagas; ergodesign; CD-ROM.
Abstract In the intersection between health, education and the control and prevention of infectious and parasitic diseases, ergodesign concepts and practices are considered fundamental elements in the generation of interfaces for learning materials in health, reflecting the relationship between user, tasks and environments. The authors conducted CD-ROM assessments based on ergodesign principles, with the aim of contributing to the access and dissemination of information relating to health and assisting in interactive learning for mid- and high-level health professionals. 21 digital materials about the two diseases, from national and international institutions, were surveyed. The materials were assessed through a checklist called the ergolist. It was found that the evaluated materials did not fully meet the evaluation criteria. The evaluation and production of information and communication technologies, such as educational and interactive CD-ROMs, based on ergodesign principles, can be a facilitating instrument in the dissemination of information and shrinking of frontiers between education and health.

Keywords health education; dengue fever and Chagas disease; ergodesign; CD-ROM. 


\title{
Introdução
}

\author{
"A arte de representar informação digital na \\ tela deveria ser vista como a forma simbólica \\ de nossa era (...). É a arte de representar zeros \\ e uns numa tela de computador"
}

(Steven Johnson, 2001, p. 4).

A experiência relatada neste artigo refere-se ao projeto de doutoramento “Disseminação de informação sobre dengue e doença de Chagas: o ergodesign no desenvolvimento e avaliação de material multimídia para educação em saúde", desenvolvido no Programa de Pós-graduação do Centro de Pesquisas René Rachou (CPqRR), da Fundação Oswaldo Cruz, em Minas Gerais ${ }^{9}$. Este projeto resultou em inúmeras colaborações, contando com a participação do Laboratório de Educação em Saúde e do Laboratório de Triatomíneos e Epidemiologia de Doença de Chagas, ambos do CPqRR; do Laboratório de Produção e Tratamento de Imagem do Instituto Oswaldo Cruz (IOC); da Editora Fiocruz; e do Laboratório de Pesquisa em Ergodesign e Usabilidade para Sistemas Humano-Tecnologia, do Centro Universitário da Cidade (LabERGUS). Neste projeto ocorreu ainda uma cooperação internacional entre a Fiocruz e a Wellcome Trust (WT), instituição filantrópica inglesa que financia e promove pesquisa em saúde humana e animal em várias partes do mundo. Conta-se também com a colaboração do Publishing Group - International Health (PGIH), área de produção e publicação de materiais digitais da Wellcome Trust, o qual vem desenvolvendo produtos de multimídia na área de saúde há mais de 11 anos, em especial CD-ROM (compact disk, read-only memory) sobre doenças infecciosas e parasitárias (Beanland et al., 2006).

O projeto de doutoramento tem como objetivo geral colaborar para o aumento de acesso ao conhecimento sobre a dengue e a doença de Chagas através de tecnologias de informação e comunicação (TIC) por meio do desenvolvimento de CD-ROM. Tem-se o intuito de explorar esta ferramenta de informação e comunicação para promover maior disseminação de informação para o controle de vetores, diagnóstico, clínica e mesmo terapêutica destas duas importantes doenças. O material desenvolvido será direcionado aos servidores de saúde de nível médio e superior10. Apresenta-se no Quadro 1 um resumo do cronograma das principais etapas desse projeto. O presente artigo relata as etapas de levantamento e de avaliação de CD-ROM de dengue e doença de Chagas disponíveis no Brasil, respectivamente a 3 e a 4 do referido projeto. 
Quadro 1

\begin{tabular}{|c|c|c|c|c|}
\hline \multicolumn{5}{|l|}{ Cronograma das principais etapas de projeto de doutoramento } \\
\hline Etapas do projeto & 2006 & 2007 & 2008 & 2009 \\
\hline \multicolumn{5}{|l|}{$\begin{array}{l}\text { 1- Tradução e adaptação do CD-ROM de dengue em inglês } \\
\text { para o português. }\end{array}$} \\
\hline \multicolumn{5}{|l|}{ 2- Avaliação do CD-ROM de dengue em português. } \\
\hline \multicolumn{5}{|l|}{ 3- Levantamento de CD-ROM sobre dengue e doença de } \\
\hline \multicolumn{5}{|l|}{ Chagas disponíveis no Brasil. } \\
\hline \multicolumn{2}{|l|}{ 4- Avaliação dos CD-ROM sobre dengue e doença de } & $=a$ & & \\
\hline \multicolumn{5}{|l|}{ Chagas disponíveis no Brasil. } \\
\hline \multicolumn{2}{|l|}{ 5- Criação do protótipo do CD-ROM de doença de Chagas. } & & 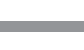 & \\
\hline \multicolumn{5}{|l|}{ 6- Avaliação do protótipo do CD-ROM de doença de } \\
\hline Chagas. & & & & \\
\hline
\end{tabular}

A primeira etapa do projeto se refere à tradução e à adaptação à realidade e ao contexto latino-americano e brasileiro de um CD-ROM produzido originalmente em inglês pela Wellcome Trust em colaboração com a UnicefUNDP-World Bank-WHO Special Programme for Research and Training in Tropical Diseases (TDR)11. Este material encontra-se em fase final de construção e será distribuído através da Editora Fiocruz e Wellcome Trust.

Na segunda etapa serão realizadas atividades de avaliação qualitativa do CD-ROM sobre dengue adaptado e traduzido para o português por meio de métodos e técnicas de ergodesign. Apesar de o CD-ROM de dengue em inglês já ter sido positivamente avaliado por alguns pesquisadores da área da saúde (Kosasih, 2005; Rico-Hesse, 2006, Leontsini, 2006), tais avaliações tiveram foco no conteúdo do $\mathrm{CD}$, onde os avaliadores eram pesquisadores da área biomédica relacionada à dengue. Porém, sabe-se que tanto a forma como o conteúdo são importantes na produção e avaliação de materiais multimídia, e é justamente nesta 'brecha' de pesquisa que a avaliação da segunda etapa será focada: na adequação do dispositivo à tarefa e ao usuário.

A terceira e a quarta etapas do projeto, foco deste artigo, constituem-se no levantamento e avaliação dos materiais digitais disponíveis no Brasil e em algumas instituições internacionais sobre dengue e doença de Chagas. Estas etapas tiveram como objetivo conhecer o estado da arte dos materiais disponíveis no Brasil para avaliar os seus pontos fortes e fracos e implementar o resultado desta avaliação na produção do CD-ROM de dengue e do protótipo de doença da Chagas. Sabe-se que antes do desenvolvimento de 
qualquer material educativo é importante uma avaliação dos tipos de materiais já existentes (Schall e Modena, 2005; Luz et al., 2003; Pimenta, 2003).

Já a quinta e a sexta etapas, desenvolvimento e avaliação do protótipo do segundo CD-ROM sobre doença de Chagas em português, serão realizadas com base nos resultados obtidos nas etapas anteriores, a fim de aproveitar os resultados de todas as avaliações no desenvolvimento de interfaces adequadas aos usuários, às tarefas realizadas e aos ambientes de uso.

\section{Exclusão digital e doenças negligenciadas: uma dupla relação de exclusão social}

As grandes endemias constituem um dos maiores desafios de saúde pública. Na história recente do Brasil, a construção do Sistema Único de Saúde (SUS) tem sido o centro deste processo, criando-se possibilidades de estratégias de controle de endemias em uma síntese que compatibilize o momento epidemiológico, a descentralização e a participação da população (Dias, 2000).

Dengue e doença de Chagas são duas doenças relevantes no cenário brasileiro que devem ser abordadas de modo integrado através de ações educativas, envolvendo a participação da comunidade. Porém, estas duas doenças ainda são categorizadas como 'doenças negligenciadas' por instituições internacionais e nacionais de saúde, gerando fortes disparidades sociais (DNDi, 2007). As doenças negligenciadas são as que afetam milhares de pessoas ao redor do mundo, mas que não dispõem de tratamentos eficazes ou adequados. Em sua maioria, são doenças tropicais infecciosas que afetam principalmente pessoas pobres, a exemplo da leishmaniose, da doença do sono, da malária e da doença de Chagas, que geram um impacto devastador sobre a humanidade.

Portanto, dengue e doença de Chagas podem ser encaradas como doenças negligenciadas. A dengue tem 'reemergido' como problema sério de saúde pública, com 100 países endêmicos no mundo. Dengue é a arbovirose mais importante no mundo, em termos de doença viral transmitida por vetores, com altas taxas de morbidade e mortalidade. Globalmente, a dengue tem uma média anual de 50-100 milhões de infecções por ano, entre os quais 15 a 20 mil são casos fatais (OMS, 2007). Sendo um dos maiores desafios de saúde pública, a dengue vem consumindo extrema energia e recursos. Sem vacina e terapêutica específica, a dengue grave demanda hospitalização em massa e pode apresentar alta letalidade. De tal modo, Dias (2000) coloca que: 
“parafraseando a Dra. Elmira Alfradique, sanitarista mineira, pode-se dizer que o dengue tem uma das melhores propostas para o SUS, colocando em risco toda a população, exigindo rede básica e cuidados secundários, requerendo medicina preventiva e não dispensando a participação de toda comunidade" (Dias, 2000, p. 288).

Já com relação à doença de Chagas, tanto pela saúde pública como pelo impacto econômico, a endemia é das mais importantes doenças parasitárias na América Latina (Miles, 2004). Estima-se que ainda existam entre 12 e 14 milhões de infectados na América Latina, com mais de 60 milhões de pessoas sob risco de transmissão, em cerca de 18 países endêmicos (OMS, 2002). Na doença de Chagas humana, tem-se uma situação de ações verticais continuadas e tecnicamente bem conduzidas que logram alto grau de controle, mesmo quando persistem as causas estruturais e econômico-sociais que residem na raiz da doença (Dias, 2000). O atual panorama brasileiro é basicamente de vigilância epidemiológica e controle dos bancos de sangue, ao lado do desafio de fornecer atenção médica e previdenciária para cerca de três milhões de infectados. Isso pressupõe, de um lado, o funcionamento dos sistemas de saúde e, de outro, a participação comunitária, no sentido de que esta vigilância deverá perdurar pelo menos por duas décadas, em especial no Nordeste (caatinga) e na Amazônia (floresta tropical) (Dias et al. 2006).

Países onde a dengue e a doença de Chagas são endêmicos, como o Brasil, necessitam desenvolver programas de controle que sejam sustentáveis e autônomos. A contínua capacitação da comunidade médica e de profissionais de saúde é um passo fundamental para a efetividade e sustentabilidade das estratégias de prevenção e controle de doenças e seus vetores. Portanto, há a necessidade de desenvolvimento de projetos que estimulem as capacidades críticas e criativas, encorajando a participação da população como um todo no processo de organização social, conhecimento do problema e produção de novas tecnologias. Identificando-se as necessidades de profissionais de saúde, busca-se incorporar inovações no campo educacional e de saúde pública, para auxiliar na prevenção e no controle da dengue e da doença de Chagas. A última edição do relatório da Organização Mundial de Saúde, o World Health Report on Knowledge for Better Health (OMS, 2004), coloca a informação em saúde como uma estratégia mundial importante de redução das disparidades globais na saúde.

Contudo, não se trata de glorificar a técnica. Não se trata de glorificar o pensamento hegemônico que sujeita o desenvolvimento humano à fabricação de ferramentas, convertendo os temas morais, éticos etc. em questões técnicas. É fundamental uma modificação estrutural nos temas de política educacional e na saúde. Como propõe Sancho: 
“(...) a solução para os problemas educacionais não está nas tecnologias da informação e da comunicação, por mais potentes que sejam os computadores para se tratar a informação ou por mais rápidas que sejam as redes de sua transmissão. O problema (...) tem profundas implicações políticas, econômicas, sociais e culturais" (Sancho, 2001, p. 12).

Junto a este contexto de doenças negligenciadas, no caso do Brasil e no da grande maioria dos países em desenvolvimento, quando associado à questão das tecnologias de informação e comunicação (TIC), o alto índice de excluídos digitais torna a situação de extrema urgência. As ações necessárias para a inclusão social envolvem diversas áreas como saúde, educação, habitação e saneamento básico, questões de cunho fortemente social. Oferecer saúde à população não é simplesmente ofertar determinados tipos de remédios ou médicos, pautados numa determinada concepção de saúde ou corpo são. Segundo Moraes (2002, p. 91), “o problema do avanço da democracia, com igualdade entre os homens e justiça social, não se resume à informação, mas, necessariamente, passa por ela". Observamos que está aumentando a distância entre os 'providos' e os 'desprovidos digitais', tanto em nível internacional quanto local.

"A chamada brecha digital preocupa não apenas porque a diferença de renda entre providos e desprovidos de tecnologia digital tende a aumentar numa época de forte inovação tecnológica, mas pela oportunidade de diminuir esta desigualdade pelas vias dos ganhos dos mais pobres. Existem poucos diagnósticos e debates no contexto brasileiro sobre o binômio inclusão/exclusão digital. (...) A discussão raramente envereda pelo acesso às tecnologias pelo lado do (...) usuário pobre (...). É preciso desenvolver tecnologias para o uso da tecnologia da informação no combate à pobreza e à desigualdade" (Néri et al., 2003, p. 4).

Desta forma, quando inter-relacionamos as TIC, em nosso caso o CDROM, e doenças infecciosas e parasitárias, especificamente dengue e doença de Chagas, surge o problema da relação entre a exclusão digital ou a falta de acesso às TIC e às chamadas doenças negligenciadas, que afetam países em desenvolvimento de uma forma avassaladora. A falta de acesso a informações relevantes, corretas e recentes ainda se constitui como uma das maiores barreiras ao conhecimento na saúde para países em desenvolvimento (Godlee et al., 2004). 


\section{Tecnologia de informação e comunicação na educação em saúde: CD-ROM e ergodesign}

É comum a afirmação de que, a partir da segunda metade do século XXI, com o desenvolvimento de tecnologias de informação e comunicação, chegou-se à 'era da informação' com transformações nas atividades econômicas, sociais e educacionais. Álvaro Vieira Pinto $(2005$, p. 7) comenta a ingenuidade desta ideologia da 'era da informação' e argumenta que "o homem não seria humano se não vivesse desde sempre nesta suposta era". A idéia de que ela foi somente inaugurada recentemente é apenas um artifício ideológico por meio do qual os grupos dominantes glorificam a sua dominação, apresentando-a como uma época áurea (Pinto, 2005), pois desde tempos pré-históricos a tecnologia e a informação são essenciais e fazem parte da cultura material humana. Assim, busca-se pensar o conceito de técnica e informação na educação em saúde como produto humano contextualizado na cultura e sociedade.

“As técnicas são imaginadas, fabricadas e reinterpretadas durante o seu uso pelos homens, como também é o próprio uso intensivo das ferramentas que constitui a humanidade enquanto tal (junto com a linguagem e as instituições complexas) (...). As tecnologias são produtos de uma sociedade e cultura. Mas a distinção traçada entre cultura (a dinâmica das representações), sociedade (as pessoas, seus laços, suas trocas, suas relações de força) e técnica (artefatos eficazes) só pode ser conceitual" (Lévy, 1999, p. 21-22).

O CD-ROM é um suporte de informação digital com leitura a laser. Tem emergido recentemente como uma das mais úteis tecnologias das últimas décadas, variando em seus aspectos na educação e em outros setores de saúde pública. Na área da saúde, são escassos os estudos relativos a este 'suporte' ou 'mídia' (Pimenta, 2006; Pimenta et al., 2007). Aliás, compartilha-se com Lévy (1999) a idéia de que se deve compreender melhor esta nova forma de 'mídia' (entendendo-se mídia como suporte ou veículo de mensagens). Para Lévy (1999):

“O dispositivo informacional qualifica a estrutura da mensagem ou o modo de relação dos elementos da informação. (...) São os novos dispositivos informacionais (mundos virtuais, informação em fluxo) e comunicacionais (comunicação todostodos) que são os maiores portadores de mutações culturais, e não o fato de que se misture o texto, a imagem e o som, como parece estar subentendido na vaga noção de multimídia" (Lévy, 1999, p. 62). 
Para alguns autores, o termo 'multimídia' significa algo que emprega diversos suportes ou diversos veículos de comunicação. Porém, a palavra refere-se a duas tendências principais de sistemas de comunicação: a multimodalidade e a integração digital. Deste modo, quem consulta um CD-ROM interativo acessa informações estruturadas em rede, passa de uma páginatela ou de uma seqüência animada para outra, indicando com um simples gesto os temas de interesse ou linhas de leitura que deseja seguir. Pode-se então considerar os CD-ROM como muito além de simples 'misturas' de mídias, mas como recursos multimodais interativos que contêm hipertextos formas mais complexas de organização e navegação de informação.

De acordo Johnson (2001, p. 4), "o trabalho de representar informação digital na tela deveria ser visto como a forma simbólica de nossa era". Assim, compreender a relevância cultural do design de interfaces no mundo de hoje é, também, uma tentativa de compreensão desta própria sociedade, de seus meios (e formas) de comunicação.

O Design é o domínio no qual se estrutura a interação entre usuário e produto, para facilitar ações efetivas. Muitas são as definições de Design, no entanto podemos considerá-lo, em linhas gerais, como:

\footnotetext{
“Uma atividade processual que consiste em determinar as propriedades formais dos objetos a serem produzidos industrialmente. Por propriedades formais, entendem-se não só as características exteriores, mas, sobretudo, as relações estruturais e funcionais que dão coerência a um objeto tanto do ponto de vista do produtor quanto do usuário" (Alvarado, 1987, p. 30).
}

Já o conceito de ergonomia, em sentido amplo, é o conjunto de conhecimentos científicos relativos ao homem e necessários à concepção de instrumentos, máquinas e dispositivos que possam ser utilizados com o máximo de conforto, segurança e eficácia (Wisner apud Fialho e Santos, 1995). Derivada do grego ergon (trabalho) e nomos (leis), para denotar a ciência do trabalho, ergonomia é uma disciplina que se estende por todos os aspectos da atividade humana (Santos, 2006). Pode-se dizer, de forma simplificada, que a ergonomia trata dos conhecimentos científicos do homem e de sua aplicação na concepção e construção de artefatos que garantam a facilitação de um desempenho global em determinado sistema, ou seja, das condições que afetam diretamente uma situação de trabalho em seus aspectos técnicos, econômicos e sociais. A ergonomia, e por conseqüência o ergodesign, parte de uma abordagem de projeto centrada no usuário.

Para Yap, Vitallis e Legg (apud Santos, 2006), o ergodesign é resultante da fusão dos focos teóricos e práticos das duas disciplinas: Ergonomia e Design. À medida que os sistemas se tornam mais complexos, fica cada vez mais difícil estabelecer diferenças entre as duas disciplinas. O ergodesign 
possui um enfoque macroergonômico criativo que busca conciliar os atributos humanos e os do sistema simultaneamente com a conceituação e desenvolvimento do projeto. Como uma tecnologia, o ergodesign tem uma orientação que o torna uma ferramenta importante, tanto no escopo quanto na eficiência da implementação da ergonomia no design e no desenvolvimento de produtos, equipamentos e sistemas. O ergodesign visa tornar as interfaces fáceis e as informações acessíveis, ou seja, centra-se na 'usabilidade' dos projetos.

Pode-se definir usabilidade como: conjunto de fatores que asseguram que os produtos sejam fáceis de usar, eficientes e agradáveis - da perspectiva do usuário. A usabilidade pode ser dividida nas seguintes metas: efetividade, eficiência, segurança, utilidade, learnability (fácil de aprender) e memorability (fácil de lembrar como se usa) (Preece et al., 2005). Também se pode entender usabilidade como a capacidade, em termos funcionais humanos, de um sistema ser usado com facilidade e com eficiência pelo usuário (Santos, 2006). Já Bastien e Scapin (1993) consideram que a usabilidade está diretamente ligada ao diálogo na interface e é a capacidade do aplicativo em permitir que o usuário alcance suas metas de interação.

A ênfase ao papel do 'usuário' ou 'público-alvo', como se costuma denominar no campo do Design e educação, respectivamente, é de extrema valia. Para tal é necessário 'conhecê-lo' através de pesquisas de usabilidade para dar 'voz' às necessidades durante todo o processo de desenvolvimento de interfaces. Trazer o usuário para dentro da equipe, para participar das tomadas de decisão e interagir com as interfaces em desenvolvimento (Moraes, 2002; Santos, 2002; Agner, 2006).

Segundo Johnson (2001), a interface é definida como:

“(...) softwares que dão forma à interação entre usuário e computador. A interface atua como uma espécie de tradutor, mediando entre duas partes, tornando uma sensível à outra (...) um computador deve também representar-se a si mesmo ao usuário numa linguagem que este compreenda" (Johnson, 2001, p. 17).

Uma interface gráfica pode ser compreendida como nova maneira de representar informação, a zona de comunicação em que se realiza a interação entre o usuário e o computador. Galvis (apud Gamez, 2004) afirma que nela estão contidos os tipos de mensagens compreensíveis pelos usuários e pelo programa, os dispositivos de entrada e saída de dados que estão disponíveis para a troca de mensagens e, ainda, as zonas de comunicação habilitadas em cada dispositivo.

São raras as iniciativas que questionem a construção e desenvolvimento de interfaces digitais na área da saúde do ponto de vista da forma e do conteúdo, pois a maioria está voltada para as questões puramente 
cognitivas e semióticas, dando ênfase somente ao conteúdo técnico ou instrucional. O ergodesign é uma atividade de caráter técnico-científico, criativo e artístico, com vistas à concepção e desenvolvimento de projetos de objetos físicos ou informacionais que equacionem sistematicamente dados tecnológicos, econômicos, sociais, culturais e estéticos, que atendam concretamente e mais eficazmente às necessidades humanas. Seu potencial é utilizado no desenvolvimento de interfaces e aplicação de conteúdos, com o objetivo de proporcionar um ambiente dinâmico e interativo para a construção de conhecimento.

\section{Metodologia e procedimentos de análise}

Foram realizadas duas grandes etapas distintas: levantamento de materiais em âmbito nacional e internacional; e seleção e avaliação dos materiais.

O levantamento foi iniciado com o envio de cartas a todas as 26 secretarias estaduais de saúde do Brasil e as 92 secretarias municipais de saúde do estado do Rio de Janeiro, as 849 de Minas Gerais e as 645 de São Paulo. Portanto, no Brasil, um total de 1.612 instituições foi abordado. Já no cenário internacional, solicitamos os mesmos materiais digitais para outras 26 instituições internacionais na área de saúde, tais como a Organização Mundial da Saúde, a Organização Pan-Americana da Saúde (Opas), Médicos sem Fronteiras, dentre outras. Como resultado, um total de 21 CD-ROM foi obtido, onde 12 são sobre doença de Chagas e nove sobre dengue.

Em uma seleção preliminar, buscou-se verificar a adequação dos materiais ao conceito de multimodalidade e de hiperdocumento, como sugerido por Lévy (1999). A maioria dos materiais não pôde ser classificada como recurso multimodal ou hiperdocumentos, pois se configuravam meramente como suportes digitais em CD-ROM para apresentações de PowerPoint e outros documentos em Word contendo apresentações científicas ou texto corrido sem ligações hipertextuais entre conteúdos. Desta forma, dos 21 materiais levantados, somente quatro caracterizavam-se como multimodais, dois sobre doença de Chagas e dois sobre dengue (CD 4, 8, 13 e 15, respectivamente), que foram os objetos da avaliação. No Quadro 2, apresenta-se a relação de todos os materiais levantados, com destaque para os que foram avaliados. 
Quadro 2

Materiais multimídia sobre dengue e doença de Chagas distribuídos no Brasil

\begin{tabular}{lccc}
\hline No & Título do material multimídia & Data & Instituição \\
\hline & & Doença de Chagas \\
\hline 1 & Manual de Chagas & 2004 & Ministério da Saúde; Secretaria de Vigilância \\
& & em Saúde; Covev - Gerência Técnica de \\
& & Controle de Vetores
\end{tabular}

2 La enfermedad de Chagas en la Argentina

2003 Ministério de Salud y Deportes; Programa

del siglo XXI

Nacional de Control de Chagas - Bolívia

3 Politicas de informacion, educacion y

2001 Ministério de Salud y Deportes - Bolívia

comunicacion para el controle de la

enfermedad de Chagas en Bolívia

4 Bibliografia brasileira sobre a doença de Chagas

Não Faculdade de Medicina do Triângulo

consta Mineiro e Fundação Nacional da Saúde; Autor: Aluísio Prata Intergubernamental de la Iniciativa Andina de Control de la Transmisión Vectorial y Transfusional de la Enfermedad de Chagas programas de control en las enfermidades transmisibles

7 Programa Nacional de Chagas IEC y Capacitacion

8 Memórias de la 2a Reunión de la Iniciativa Intergubernamental de Vigilancia y Prevención de la Enfermedad de Chagas en la Amazonia
$5 \quad$ V Reunión de la Comisión

6 Taller: impacto de descentralización de los

2004 Organização Pan-Americana da Saúde (Opas) / Organização Mundial da Saúde (OMS) e República del Peru

2005 Organização Pan-Americana da Saúde (Opas) / Organização Mundial da Saúde (OMS)

2001 Ministério de Salud y Deportes de Bolívia; Escudo Epidemiológico

2006 Centro Internacional de Investigaciones para el Desarrollo (IDRC); Iniciativa programática enfoques ecosistémicos para la salud humana (Ecosalud); Organização Pan-Americana da Saúde (Opas) / Organização Mundial da Saúde (OMS); Centro de Pesquisa Leônidas \& Maria Deane (CPqL\&MD), Fiocruz, Amazônia

9 Informe preliminar linea base y monitoreo

2003 Ministério de Salud y Deportes; Dirección entomologico General de Control y Prevención de Enfermedades; Programa Nacional de Enfermedades Transmitidas por Vectores; Programa Nacional de Control de Chagas

10 Faire Face la Maladie de Chagas en Partant 2006 Université de Genéve des Conceptions des Populations Concernées 
continuação - Quadro 2

\begin{tabular}{|c|c|c|c|}
\hline No & Título do material multimídia & Data & Instituição \\
\hline 11 & $\begin{array}{l}\text { Consulta técnica regional Opas/MSF sobre } \\
\text { organización y estructura de la atención } \\
\text { médica del enfermo e infectado por } \\
\text { Trypanosoma cruzi / enfermedad de } \\
\text { Chagas }\end{array}$ & 2005 & $\begin{array}{l}\text { Organização Pan-Americana da Saúde } \\
\text { (Opas)/Organização Mundial da Saúde (OMS); } \\
\text { Médicos sem Fronteiras (MSF) }\end{array}$ \\
\hline 12 & $\begin{array}{l}\text { Núcleo de controle de endemias } \\
\text { transmissíveis por vetores Nuend }\end{array}$ & 2004 & $\begin{array}{l}\text { Governo do Estado do Ceará; Secretaria de } \\
\text { Saúde (Sesa); Coordenadoria de } \\
\text { Desenvolvimento, Apoio e Atenção à Saúde } \\
\text { (Codas) }\end{array}$ \\
\hline & & Dengue & \\
\hline 13 & Dengue & $\begin{array}{l}\text { Não } \\
\text { consta }\end{array}$ & $\begin{array}{l}\text { Prefeitura do Município de Itapevi, São } \\
\text { Paulo; Secretaria de Higiene e Saúde }\end{array}$ \\
\hline 14 & $\begin{array}{l}\text { CDC Clinical Manifestations and } \\
\text { Epidemiology }\end{array}$ & $\begin{array}{c}\text { Não } \\
\text { consta }\end{array}$ & $\begin{array}{l}\text { Center for Disease Control and Prevention } \\
\text { (CDC) }\end{array}$ \\
\hline 15 & $\begin{array}{l}\text { IDRD - Brasil: } 19 \text { anos de cooperação } \\
\text { científica no estudo dos arbovírus }\end{array}$ & 2001 & $\begin{array}{l}\text { Institut de Recherche pour le } \\
\text { développement (IDRD); Instituto Evandro } \\
\text { Chagas de Belém; Instituto de Saúde do } \\
\text { Distrito Federal, Brasília. }\end{array}$ \\
\hline 16 & $\begin{array}{l}\text { Campanha de combate à dengue "Bota } \\
\text { Fora Dengue" }\end{array}$ & $\begin{array}{l}\text { Não } \\
\text { consta }\end{array}$ & $\begin{array}{l}\text { Secretaria Municipal de Saúde de Itabirito, } \\
\text { Minas Gerais }\end{array}$ \\
\hline 17 & Jogos Dengue & $\begin{array}{l}\text { Não } \\
\text { consta }\end{array}$ & $\begin{array}{l}\text { Sascha Wolter (www.saschawolter.de) - } \\
\text { freelancer }\end{array}$ \\
\hline 18 & Palhaço Figurinha e dengue & $\begin{array}{c}\text { Não } \\
\text { consta }\end{array}$ & Prefeitura de Araxá, Minas Gerais \\
\hline 19 & Fotos dengue & $\begin{array}{c}\text { Não } \\
\text { consta }\end{array}$ & Prefeitura de São João Del Rey, Minas Gerais \\
\hline 20 & Campanha Dengue & $\begin{array}{l}2005 / \\
2006\end{array}$ & $\begin{array}{l}\text { Prefeitura da cidade de São Paulo; } \\
\text { Coordenação de Vigilância em Saúde (Covisa) }\end{array}$ \\
\hline 21 & $\begin{array}{l}\text { Apresentações Dengue e doença de } \\
\text { Chagas }\end{array}$ & $\begin{array}{c}\text { Não } \\
\text { consta }\end{array}$ & $\begin{array}{l}\text { Prefeitura Municipal de Botelhos, Minas } \\
\text { Gerais }\end{array}$ \\
\hline
\end{tabular}

Nota: Os materiais analisados neste estudo estão em negrito.

Com o objetivo de levantar índices de aplicabilidade, conformidade e fornecer indicações sobre a usabilidade do sistema, utilizou-se para avaliação o ergolist, uma ferramenta para inspeção de usabilidade de interfaces baseada em critérios ergonômicos. É uma ferramenta gratuita, já validada, e 
está disponível na internet no endereço www.labiutil.inf.ufsc.br/ergolist/ index.html. Os critérios ergonômicos do ergolist são: concisão, mensagens de erro, flexibilidade, legibilidade, significados, proteção contra erros, agrupamento por formato, experiência do usuário, presteza, controle do usuário, correção de erros, consistência, agrupamento por localização, densidade informacional, feedback, compatibilidade, ações explícitas e ações mínimas.

Cada um dos quatro materiais selecionados foi avaliado individualmente a partir de cada um dos critérios listados acima.

\section{Resultados preliminares}

Os resultados demonstram que a maioria dos materiais apresentou um alto índice de não conformidade aos critérios utilizados na avaliação. Este fato é decorrente da não aplicação de conceitos de ergodesign e usabilidade durante o projeto, o que corrobora a afirmação de que a preocupação se restringiu à elaboração de conteúdo, em detrimento da interface, o que causa dificuldades de uso por parte do usuário final. Dentre os problemas verificados, destacam-se a dificuldade de navegação, dificuldade para localizar conteúdo e possibilidade de sentir-se perdido durante o uso.

No Gráfico 1, verificam-se todos os critérios de usabilidade avaliados de acordo com questões de não conformidade para todos os CD-ROM selecionados. A região hachurada indica valores de não conformidade acima de 50\%. Esses valores são considerados como inadequados ou mal utilizados nos CD-ROM.

Gráfico 1

Critérios de usabilidade relacionados à não conformidade dos CD-ROM avaliados

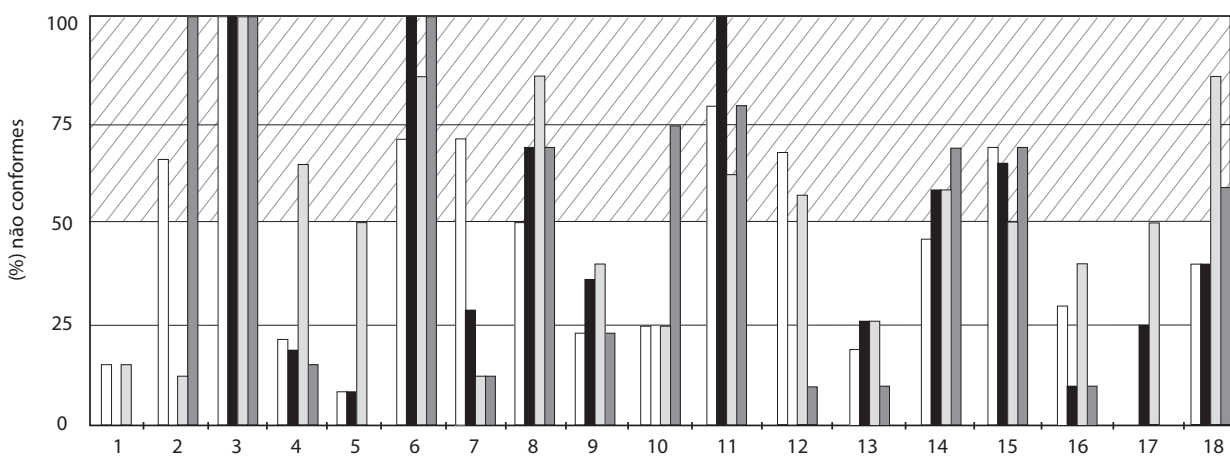

Critérios de usabilidade: $\square$ CD-04 $\square$ CD-08 $\square$ CD-13 $\square$ CD-15 
A linguagem visual é um grande desafio na construção de material educativo. Quanto mais adaptado à interface for para o usuário, mais este saberá navegar dentro dela. Neste quesito, alguns materiais foram tentativas desta adaptação, pois alguns materiais tinham apresentação visual semelhante à web, mais especificamente a sites científicos de pesquisa. Porém, a falta de usabilidade dificultou muito o acesso a informações pertinentes.

Com relação à consistência, devem-se usar palavras de forma padronizada tanto no conteúdo quanto na forma e, em geral, este fato foi observado na metade dos materiais avaliados. Apesar de tal padronização, com relação específica ao conteúdo, muitas vezes se viam misturadas questões de ordem prática e científica com conceitos do 'senso comum' que, muitas vezes, 'banalizavam' a problematização sobre as doenças.

Por exemplo, o CD-4 apresenta conteúdo em telas de tamanhos diferentes. Além de ter uma forma de apresentação diferente das iniciais, leva o usuário a não identificar a totalidade do material. O tipo de letra adotado é de difícil leitura, principalmente quando utilizado em tamanhos menores. Com relação às cores, foram consideradas adequadas: telas iniciais verdes e fundo branco com letras pretas na listagem de trabalhos, o que facilita a leitura e a impressão dos textos.

O CD-8 já se apresenta em formato de web, mostrando telas com cores neutras e tela dimensionada à largura máxima do sistema operacional, o que se torna prejudicial aos textos, que ficam com linhas muito longas. Já na tela principal, a tela do menu, misturam-se conteúdos de uma secção intitulada 'Trabajos', que possui anexos e poderiam estar em uma seção específica, pois existe diferenciação nos seus conteúdos. Somente o CD-8 continha programa auto-executável, o que permite que o material seja apresentado automaticamente após a inserção do CD-ROM no computador. Se o material não tiver este recurso, é necessário que o usuário encontre uma maneira de acessar o conteúdo, como observado no $\mathrm{CD}-13$. Neste $\mathrm{CD}$, para ter o conteúdo visualizado plenamente, é necessário um recurso adicional não fornecido com o CD-ROM e nem há indicação de como obtê-lo.

A maioria dos materiais não continha mensagem de erro indicando ao usuário como proceder em caso de mau uso ou subutilização do material. Somente o CD-8 continha um local para busca de ajuda que utilizou o programa Macromedia Flash, onde se explica em áudio como o conteúdo do $\mathrm{CD}$ está exposto e organizado. Esta montagem por esse programa está bem feita, porém, se o usuário não tiver uma saída de áudio, fica incompreensível. Desta forma, é aconselhável que, junto com o áudio, também se inclua 
algum tipo de texto simultâneo.

Concisão também é outro critério importante que não foi bem aplicado na maioria dos materiais. Em geral, as interfaces não auxiliaram na organização do conteúdo, fazendo com que o usuário se perca com mais facilidade. A maioria dos conteúdos se assemelhou a textos impressos, onde a informação é disposta linearmente, com excessivo uso de textos, enchendo a tela com dados e imagens nem sempre relevantes ao tema.

O Quadro 3 apresenta um resumo de todos os critérios ergonômicos do ergolist que foram avaliados com uma porcentagem acima de $50 \%$, ou seja, critérios que tiveram uma porcentagem mais alta de conformidade das avaliações de usabilidade. Os critérios em negrito não estiveram presentes nos materiais analisados.

Quadro 3

\begin{tabular}{|c|c|c|c|c|c|}
\hline \multicolumn{6}{|c|}{ Critérios ergonômicos de conformidade dos CD-ROM avaliados } \\
\hline$\#$ & Critérios & $C D n^{\circ} 4$ & $C D n^{\circ} 8$ & $C D n^{\circ} 13$ & $C D n^{\circ} 15$ \\
\hline 1 & Concisão & $x$ & $\mathrm{x}$ & $x$ & $x$ \\
\hline 2 & Mensagens de erro & - & $x$ & $x$ & - \\
\hline 3 & Flexibilidade & - & - & - & - \\
\hline 4 & Legibilidade & $x$ & $x$ & - & $x$ \\
\hline 5 & Significados & $x$ & $x$ & - & $x$ \\
\hline 6 & Proteção contra erros & - & - & - & - \\
\hline 7 & Agrupamento por formato & - & $x$ & $x$ & $x$ \\
\hline 8 & Experiência do usuário & - & ! & ! & ! \\
\hline 9 & Presteza & $x$ & $x$ & $x$ & $x$ \\
\hline 10 & Controle do usuário & $x$ & $x$ & $x$ & - \\
\hline 11 & Correção de erros & - & - & - & - \\
\hline 12 & Consistência & - & $x$ & - & $x$ \\
\hline 13 & Agrupamento por localização & $x$ & $x$ & $x$ & $x$ \\
\hline 14 & Densidade informacional & $x$ & ! & - & ! \\
\hline
\end{tabular}


continuação - Quadro 3

\begin{tabular}{|c|c|c|c|c|c|}
\hline \# & Critérios & $C D n^{\circ} 4$ & $C D n^{\circ} 8$ & $C D n^{\circ} 13$ & $C D n^{\circ} 15$ \\
\hline 15 & Feedback & - & ! & a & - \\
\hline 16 & Compatibilidade & $x$ & $x$ & $x$ & $x$ \\
\hline 17 & Ações explícitas & $x$ & $x$ & - & $x$ \\
\hline 18 & Ações mínimas & $x$ & $x$ & - & - \\
\hline
\end{tabular}

X Não atendeu aos critérios ergonômicos

- Atendeu aos critérios ergonômicos

Desta forma, observou-se que os critérios de flexibilidade, proteção contra erros, experiência do usuário e feedback tiveram uma avaliação abaixo de $50 \%$ e, portanto, sequer foram considerados no desenvolvimento dos materiais avaliados. Estes critérios são importantes para garantia da usabilidade dos materiais, em especial o critério com relação à experiência do usuário.

\section{Abordagens na construção de materiais interativos: para além do monólogo}

A avaliação de materiais digitais e interativos em saúde demanda um estudo mais aprofundado da concepção, utilização, produção de interfaces abarcando os três principais componentes: usuários, tarefas e contextos (ambiente). Cada produto ou obra requer uma análise do tipo de relação desejada entre os critérios de usabilidade e interface. Assim, através deste estudo, pôde-se observar que: os materiais sobre dengue e doença de Chagas distribuídos no Brasil não são desenvolvidos a partir de uma abordagem centrada no usuário, dificultando sua utilização; e a aplicação de critérios de ergodesign e de usabilidade na produção de material multimídia possibilita o melhor exercício do agente de saúde como disseminador de informações sobre dengue e doença de Chagas.

Análises de materiais digitais distribuídos no Brasil mostram inúmeros conceitos e formas de tratamento do conteúdo, já constatados em outros materiais educativos, tais como vídeos e impressos. Como no caso das interfaces que se assemelharam a 'aulas' ou 'palestras', trata-se o conteúdo de maneira uniforme, independentemente do formato ou meio no qual este se veicula. Esta característica também foi observada por Pimenta $(2003 ; 2007)$ 
no caso de vídeos educativos sobre leishmaniose.

Na maioria dos materiais educativos em saúde pública, a transferência de informação se dá tipicamente em forma de 'monólogo', onde a informação flui em uma direção única - do 'detentor de informações' (geralmente países desenvolvidos) para 'recipientes' (países em desenvolvimento), lembrando a 'educação bancária' de Paulo Freire (1987), que há muito tempo tem sido alvo de críticas. Encontramos, portanto, uma necessidade de se ir além das práticas tradicionais de transferência de informação (monólogo unilateral), para um intercâmbio de informações (comunicação dialógica). Ao se produzir o conteúdo do CD-ROM de dengue e CD-ROM de doença de Chagas no Brasil, busca-se desenvolver um produto a partir de uma abordagem de projeto centrado no usuário e que reflita melhor a realidade do local onde estas duas doenças são endêmicas, com vistas a facilitar a dialogicidade do processo comunicacional.

Pode-se concluir que os materiais digitais necessitam ser produzidos a partir das necessidades do público usuário, levando-se sempre em conta o contexto e a finalidade de uso. Chama-se a atenção para a necessidade de estudos que avaliem e compreendam a relação existente entre materiais educativos em saúde e a utilização e criação de interfaces digitais, evidenciando a necessidade de estudos qualitativos sobre saúde, educação e ergodesign.

As instituições voltadas para a disseminação da informação encontram-se em um momento de questionamento tanto sobre as práticas estabelecidas quanto sobre as novas possibilidades criadas pela introdução das TIC. A apreensão das múltiplas facetas deste modelo é condição fundamental para a condução de projetos de inserção dos usuários neste cenário. O debate sobre a disseminação da informação no contexto das tecnologias na educação em saúde, em especial na divulgação de informações sobre doenças negligenciadas como a dengue e doença de Chagas, deve ser apresentado não como uma doutrina rígida, mas em seu processo e no desenvolvimento de suas idéias.

Busca-se, portanto, estabelecer a aplicação de critérios de ergodesign e usabilidade na produção de material multimídia cuja utilização possibilite ao agente de saúde atuar efetivamente como disseminador de informações sobre dengue e doença de Chagas. Desta forma, para poder se adotar uma posição crítica e de valor e não só de consumo indiscriminado, é necessário entender as chaves das linguagens das TIC, ter capacidade para saber aprender, critério para selecionar e situar a informação e um mínimo de entendimento básico para dar-lhe sentido e convertê-la em conhecimento pessoal, profissional e social. 


\section{Agradecimentos}

Agradecemos à Fundação de Amparo à Pesquisa do Estado de Minas Gerais (Fapemig), Fundação Oswaldo Cruz (Fiocruz) e Wellcome Trust, que apoiaram o estudo que deu origem a este artigo.

\section{Notas}

1 Pesquisadora do Laboratório de Educação em Saúde, do Centro de Pesquisas René Rachou, da Fundação Oswaldo Cruz (CPqRR/Fiocruz), Belo Horizonte, Brasil. Doutoranda em Ciências da Saúde pelo Programa de Pós-Graduação em Ciências da Saúde do CPqRR. $<$ nacif@cpqrr.fiocruz.br> Correspondência: Laboratório de Educação em Saúde, Centro de Pesquisas René Rachou, Fundação Oswaldo Cruz, Av. Augusto de Lima, 1.715, Barro Preto, Belo Horizonte, Minas Gerais, Brasil, CEP 30190-002.

2 Progamadora visual do Instituto Oswaldo Cruz, da Fundação Oswaldo Cruz (IOC/Fiocruz), Rio de Janeiro, Brasil. Graduada em Comunicação Visual pelo Centro Universitário da Cidade (UniverCidade). Especialista em Design de Interfaces pelo Centro Universitário Carioca (UniCarioca). < heloisa@ioc.fiocruz.br>

3 Graduando em Arquitetura pela Universidade Federal de Minas Gerais (UFMG), Belo Horizonte, Brasil. <manucasinuca@hotmail.com>

4 Graduanda em Design Gráfico pela Universidade do Estado de Minas Gerais (UEMG), Belo Horizonte, Brasil. <patirezende@gmail.com>

5 Graduação em Letras pela Universidade Federal de Minas Gerais (UFMG), Belo Horizonte, Brasil. <giuliana_fs@hotmail.com>

6 Pesquisador titular do Centro de Pesquisas René Rachou, da Fundação Oswaldo Cruz (CPqRR/Fiocruz), Belo Horizonte, Brasil. Doutor em Medicina Tropical pela UFMG. <jcpdias@cpqrr.fiocruz.br>

7 Pesquisadora titular do Centro de Pesquisas René Rachou, da Fundação Oswaldo Cruz (CPqRR/Fiocruz), Belo Horizonte, Brasil. Doutora em Educação pela Pontifícia Universidade Católica do Rio de Janeiro (PUC-RJ). <vtschall@cpqrr.fiocruz.br>

8 Professor adjunto do Centro Universitário da Cidade (UniverCidade), Rio de Janeiro, Brasil. Doutor em Design pela Pontifícia Universidade Católica do Rio de Janeiro (PUC-RJ). <contato@robsonsantos.com>

${ }^{9}$ Este artigo foi extraído da tese de doutorado intitulada “Disseminação de informação sobre dengue e doença de Chagas: o ergodesign no desenvolvimento e avaliação de material multimídia para educação em saúde", de Denise Nacif Pimenta. 
10 Atualmente, a Fiocruz não desenvolve uma produção ampla de CD-ROM. A maioria de sua produção é de origem externa à instituição através de contratação de empresas privadas. Eymard M. Vasconcelos aponta o potencial perigo de tais produções: “É preciso superar a atual situação, em que as grandes campanhas educativas em saúde são organizadas por grandes empresas de comunicação muito pouco articuladas com o cotidiano de relação entre os profissionais de saúde e a população" (Vasconcelos, 2004, p. 70).

11 Denise Nacif Pimenta fez parte do OMS/TDR Career Development Fellowship: Interactive Learning Production. Durante este fellowship na Wellcome Trust, Londres (2003-2005), participou do desenvolvimento do primeiro CD-ROM sobre dengue, produzido originalmente em inglês, pela Wellcome Trust - Publishing Group International Health (PGIH) em colaboração com a Unicef-UNDP-World Bank-WHO Special Programme for Research and Training in Tropical Diseases (TDR). Para maiores informações e pedidos do CD-ROM em inglês, acesse: www.wellcome.ac.uk/node5824.html.

\section{Referências}

AGNER, Luiz. Ergodesign e arquitetura da informação: trabalhando com o usuário. Rio de Janeiro: Quartet, 2006.

ALVARADO, Daisy Valle Machado Peccinini. Novas figurações, novo realismo e nova objetividade. Brasil anos 60. Tese de doutorado. Escola de Comunicações e Artes da USP. São Paulo: ECA/USP, 1987.

BASTIEN, J. M. Christian; SCAPIN, Dominique. Ergonomics Criteria for the Evaluation of Human-Computer Interfaces. Relatório de Pesquisa $\mathrm{n}^{\circ}$ 156. França: Institut National de Recherche en Informatique et en Automatique. Rocquencourt (Inria), 1993.

BEANLAND, Tim J. et al. Multimedia materials for education, training, and advocacy in international health: experiences with the Schistosomiasis Control Initiative CDROM. Memórias do Instituto Oswaldo Cruz, v. 101, supl. 1, p. 87-90, 2006.

DIAS, João C. P. Participação, descentralização e controle de endemias no Brasil. In: BARATA, Rita B.; BRICEÑO-LEÓN, Roberto
(Org.). Doenças endêmicas: abordagens sociais, culturais e comportamentais. Rio de Janeiro: Editora Fiocruz, p. 269-297, 2000.

DIAS, João C. P.; CARVALHO, Sílvia H.; PIMENTA, Denise N. A doença de Chagas: legitimamente brasileira. In: TOLEDO JÚNIOR, Antônio C. C. (Org.). Pragas e epidemias: histórias de doenças infecciosas. Belo Horizonte: Folium, p. 45-60, 2006.

DNDi (Drugs for Neglected Disease Iniciative). Dados sobre doenças negligenciadas. Genebra, 2007. Disponível em: $<$ www.dndi.org.br/Portugues/doencas_ne gligenciadas.aspx $>$. Acesso em: 29 nov. 2007.

FIALHO, Francisco; SANTOS, Neri. Manual de análise ergonômica no trabalho. Curitiba: Gênesis, 1995.

FREIRE, Paulo. Pedagogia do oprimido. Rio de Janeiro: Paz e Terra, 1987.

GAMEZ, Luciano. A construção da coerência em cenários pedagógicos online: uma metodologia para apoiar a transformação de 
cursos presenciais que migram para a modalidade de educação a distância. Tese de doutorado. Florianópolis: UFSC, 2004.

GODLEE, Fiona et al. Can we achieve health information for all by 2015?. Londres. The Lancet, v. 364, n. 9.430, p. 295300, 2004.

JOHNSON, Steven. A cultura da interface. Rio de Janeiro: Jorge Zahar Editor, 2001.

KOSASIH, Herman. ADBI Institute: Sharing Development Knowledge about Asia and the Pacific. Genebra, 2005. Disponível em: $<$ www.who.int/rpc/meetings/en/WR2004 AnnotatedOutline.pdf.> Acesso em: 13 jan. 2007.

LEONTSINI, Elli. Software Review. Dengue. Annals of Tropical Medicine \& Parasitology, v. 100, n. 1, p. 91-93, 2006.

LÉVY, Pierre. Cibercultura. São Paulo: Editora $34,1999$.

LUZ, Zélia M. P. et al. Evaluation of informative materials on leishmaniasis distributed in Brazil: criteria and basis for the production and improvement of health education materials. Cadernos de Saúde Pública, v. 19, n. 2, p. 561-569, 2003.

MILES, Michael A. The discovery of Chagas Disease: progress and prejudice. Infect Dis Clin N Am, v. 18, p. 247-260, 2004.

MORAES, Anamaria de (Org). Design e avaliação de interface: ergodesign e interação humano-computador. Rio de Janeiro: iUsEr, 2002.

MORAES, Ilara Hämmerli S. de. Política, tecnologia e informação em saúde. Salvador: Casa da Qualidade Editora, 2002.

NÉRI, Marcelo et al. A. Lei de Moore e políticas de inclusão digital. Revista Inteligência Empresarial, n. 14, jan., 2003.
ORGANIZAÇÃO MUNDIAL DA SAÚDE (OMS). 2007. Dengue Fact Sheet. Disponível em: <www.who.int/mediacentre/ factsheets/fs117/en/>. Acesso em: 5 jun. 2007.

World Report on knowledge for better health. Geneva, 2004: World Health Organization. Disponível em: $<$ www.who.int/rpc/meetings/en/WR2004 AnnotatedOutline.pdf $>$. Acesso em: 15 abr. 2005.

Control of Chagas disease. Geneva: World Health Organization. Technical Report Series, 905, 2002.

PIMENTA, Denise N. Can the North learn from developing countries: question or affirmation? Global Forum for Health Research, Young Voices. Genebra: Research for Health, 2007, v. 1, p. 102-105. Disponível em: <www.globalforumhealth.org/Site/002 _What $\% 20$ we $\% 20$ do/005_Publications/ 012 _Young $\% 20$ Voices $\% 20$ in $\%$ 20Researc $\mathrm{h} \% 20$ for $\% 20$ Health.php $>$. Acesso em: 2 dez. 2007.

As leishmanioses e suas representações: interação da linguagem do documentário com os saberes e práticas populares. Dissertação de mestrado. Rio de Janeiro: Núcleo de Tecnologia Educacional para Saúde, UFRJ, 2003.

PIMENTA, Denise N.; LEANDRO, Anita; SCHALL, Virgínia T. A estética do grotesco e a produção audiovisual para a educação em saúde: segregação ou empatia? O caso das leishmanioses no Brasil. Cadernos de Saúde Pública, v. 23, n. 5, p. 1161-1171, 2007.

desenvolvimento e avaliação de materiais educativos sobre saúde: abordagens sóciohistóricas e contribuições da antropologia visual. In: MONTEIRO, Simone; VARGAS, Eliane (Orgs.). Educação, comunicação $e$ 
tecnologia educacional: interfaces com o campo da saúde. Rio de Janeiro: Editora Fiocruz, p. 87-112, 2006.

PINTO, Álvaro Vieira. O conceito de tecnologia. Rio de Janeiro: Contraponto, 2005.

PREECE, Jennifer; ROGERS, Yvonne; SHARP, Helen. Design de interação: além da interação homem-computador. Porto Alegre: Bookman, 2005.

RICO-HESSE, Rebeca. CD-ROM Review. Dengue: Topics in International Health CDROM. American Journal of Tropical Medicine and Hygine, v. 75, n. 5, p. 1021-1022, 2006.

SANCHO, Juana Maria (Org.). Para uma tecnologia educacional. Porto Alegre: ArtMed, 2001.

SANTOS, Robson. Usabilidade de interfaces para sistemas de recuperação de informação na web. Dissertação de doutorado. Rio de
Janeiro: Departamento de Artes e Design, PUC-RJ, 2006.

SANTOS, Robson. Abordagem heurística para avaliação da usabilidade de interfaces. In: MORAES, Anamaria (Org). Design e avaliação de interface: ergodesign e interação humano-computador. Rio de Janeiro: iUsEr, p.59-88, 2002.

SCHALL, Virgínia T.; MODENA, Celina M. As novas tecnologias de informação e comunicação em educação em saúde. In: MINAYO, Maria C. S.; COIMBRA Jr., Carlos E. A. (Orgs.). Críticas e atuantes: ciências sociais e humanas em saúde na América Latina. Rio de Janeiro: Editora Fiocruz, p. 245-255, 2005.

VASCONCELOS, Eymard Mourão. Educação popular: de uma prática alternativa a uma estratégia de gestão participativa das políticas de saúde. Physis: Revista de Saúde Coletiva, v. 14, n. 1, p. 67-83, 2004.

Recebido em 08/05/2007

Aprovado em 08/02/2008 